\title{
Sublinear operators with rough kernel generated by Calderón-Zygmund operators and their commutators on generalized local Morrey spaces
}

\author{
Aydin S Balakishiyev' ${ }^{1}$ Vagif S Guliyev ${ }^{2,3}$, Ferit Gurbuz ${ }^{4}$ and Ayhan Serbetci ${ }^{*}$
}

${ }^{*}$ Correspondence: serbetci@ankara.edu.tr

${ }^{4}$ Department of Mathematics,

Ankara University, Ankara, Turkey

Full list of author information is

available at the end of the article

\begin{abstract}
In this paper, we will study the boundedness of a large class of sublinear operators with rough kernel $T_{\Omega}$ on the generalized local Morrey spaces $L M_{p, \varphi}^{\left\{x_{0}\right\}}$, for $s^{\prime} \leq p, p \neq 1$ or $p<s$, where $\Omega \in L_{s}\left(S^{n-1}\right)$ with $s>1$ are homogeneous of degree zero. In the case when $b \in L C_{p, \lambda}^{\left\{x_{0}\right\}}$ is a local Campanato spaces, $1<p<\infty$, and $T_{\Omega, b}$ be is a sublinear commutator operator, we find the sufficient conditions on the pair $\left(\varphi_{1}, \varphi_{2}\right)$ which ensures the boundedness of the operator $T_{\Omega, b}$ from one generalized local Morrey space $L M_{p, \varphi_{1}}^{\left\{x_{0}\right\}}$ to another $L M_{p, \varphi_{2}}^{\left\{x_{0}\right\}}$. In all cases the conditions for the boundedness of $T_{\Omega}$ are given in terms of Zygmund-type integral inequalities on $\left(\varphi_{1}, \varphi_{2}\right)$, which do not make any assumptions on the monotonicity of $\varphi_{1}, \varphi_{2}$ in $r$. Conditions of these theorems are satisfied by many important operators in analysis, in particular pseudo-differential operators, Littlewood-Paley operators, Marcinkiewicz operators, and Bochner-Riesz operators.
\end{abstract}

MSC: $42 \mathrm{~B} 20 ; 42 \mathrm{~B} 25 ; 42 \mathrm{~B} 35$

Keywords: sublinear operator; Calderón-Zygmund operator; rough kernel; generalized local Morrey space; commutator; local Campanato space

\section{Introduction}

For $x \in \mathbb{R}^{n}$ and $r>0$, let $B(x, r)$ denote the open ball centered at $x$ of radius $r,{ }^{\mathrm{C}} B(x, r)$ denote its complement and $|B(x, r)|$ is the Lebesgue measure of the ball $B(x, r)$. Suppose that $S^{n-1}$ is the unit sphere in $\mathbb{R}^{n}(n \geq 2)$ equipped with the normalized Lebesgue measure $d \sigma$.

Let $\Omega \in L_{s}\left(S^{n-1}\right)$ with $1<s \leq \infty$ be homogeneous of degree zero. Suppose that $T_{\Omega}$ represents a linear or a sublinear operator, which satisfies, for any $f \in L_{1}\left(\mathbb{R}^{n}\right)$ with compact support and $x \notin \operatorname{supp} f$,

$$
\left|T_{\Omega} f(x)\right| \leq c_{0} \int_{\mathbb{R}^{n}} \frac{|\Omega(x-y)|}{|x-y|^{n}}|f(y)| d y
$$

where $c_{0}$ is independent of $f$ and $x$.

\section{Springer}

C) 2015 Balakishiyev et al: licensee Springer. This is an Open Access article distributed under the terms of the Creative Commons Attribution License (http://creativecommons.org/licenses/by/4.0), which permits unrestricted use, distribution, and reproduction in any medium, provided the original work is properly credited. 
For a function $b$, suppose that the commutator operator $T_{\Omega, b}$ represents a linear or a sublinear operator, which satisfies, for any $f \in L_{1}\left(\mathbb{R}^{n}\right)$ with compact support and $x \notin \operatorname{supp} f$,

$$
\left|T_{\Omega, b} f(x)\right| \leq c_{0} \int_{\mathbb{R}^{n}}|b(x)-b(y)| \frac{|\Omega(x-y)|}{|x-y|^{n}}|f(y)| d y,
$$

where $c_{0}$ is independent of $f$ and $x$.

We point out that the condition (1.1) in the case $\Omega \equiv 1$ was first introduced by Soria and Weiss in [1]. The condition (1.1) is satisfied by many interesting operators in harmonic analysis, such as the Calderón-Zygmund operators, Carleson maximal operators, HardyLittlewood maximal operators, C Fefferman singular multipliers, R Fefferman singular integrals, Ricci-Stein oscillatory singular integrals, the Bochner-Riesz means, and so on (see $[1,2]$ for details).

Let $\Omega \in L_{s}\left(S^{n-1}\right)$ with $1<s \leq \infty$ be homogeneous of degree zero and satisfy the cancelation condition

$$
\int_{S^{n-1}} \Omega\left(x^{\prime}\right) d \sigma\left(x^{\prime}\right)=0
$$

where $x^{\prime}=x /|x|$ for any $x \neq 0$. The homogeneous singular integral operator $\bar{T}_{\Omega}$ defined by

$$
\bar{T}_{\Omega} f(x)=p \cdot v \cdot \int_{\mathbb{R}^{n}} \frac{\Omega(x-y)}{|x-y|^{n}} f(y) d y
$$

satisfies the condition (1.1).

It is obvious that when $\Omega \equiv 1, \bar{T}_{\Omega}$ is the singular integral operator $\bar{T}$.

Theorem A ([3]) Suppose that $1 \leq p<\infty, \Omega \in L_{s}\left(S^{n-1}\right), s>1$, is homogeneous of degree zero and has mean value zero on $S^{n-1}$. If $s^{\prime} \leq p, p \neq 1$ or $p<s$, then the operator $\bar{T}_{\Omega}$ is bounded on $L_{p}\left(\mathbb{R}^{n}\right)$. Also the operator $\bar{T}_{\Omega}$ is bounded from $L_{1}\left(\mathbb{R}^{n}\right)$ to $W L_{1}\left(\mathbb{R}^{n}\right)$.

Let $b$ be a locally integrable function on $\mathbb{R}^{n}$, then we shall define the commutators generated by singular integral operators with rough kernels and $b$ as follows:

$$
\left[b, \bar{T}_{\Omega}\right] f(x) \equiv b(x) \bar{T}_{\Omega} f_{1}(x)-\bar{T}_{\Omega}(b f)(x)=p . v . \int_{\mathbb{R}^{n}}[b(x)-b(y)] \frac{\Omega(x-y)}{|x-y|^{n}} f(y) d y .
$$

Theorem B ([3]) Suppose that $\Omega \in L_{s}\left(S^{n-1}\right), s>1$, is homogeneous of degree zero and has mean value zero on $S^{n-1}$. Let $1<p<\infty$ and $b \in B M O\left(\mathbb{R}^{n}\right)$. If $s^{\prime} \leq p$ or $p<s$, then the commutator operator $\left[b, \bar{T}_{\Omega}\right]$ is bounded on $L_{p}\left(\mathbb{R}^{n}\right)$.

The classical Morrey spaces $M_{p, \lambda}$ were first introduced by Morrey in [4] to study the local behavior of solutions to second order elliptic partial differential equations. For the boundedness of the Hardy-Littlewood maximal operator, the fractional integral operator and the Calderón-Zygmund singular integral operator on these spaces, we refer the readers to [5-7]. For the properties and applications of classical Morrey spaces, see [8-11] and references therein. The generalized Morrey spaces $M_{p, \varphi}$ are obtained by replacing $r^{\lambda}$ by a function $\varphi(r)$ in the definition of the Morrey space. During the last decades various classi- 
cal operators, such as maximal, singular, and potential operators, were widely investigated in both in classical and generalized Morrey spaces.

In this paper, we prove the boundedness of the operators $T_{\Omega}$ from one generalized local Morrey space $L M_{p, \varphi_{1}}^{\left\{x_{0}\right\}}$ to another $L M_{p, \varphi_{2}}^{\left\{x_{0}\right\}}, 1<p<\infty$, and from the space $L M_{1, \varphi_{1}}^{\left\{x_{0}\right\}}$ to the weak space $W L M_{1, \varphi_{2}}^{\left\{x_{0}\right\}}$. In the case $b \in L C_{p_{2}, \lambda}^{\left\{x_{0}\right\}}$, we find the sufficient conditions on the pair $\left(\varphi_{1}, \varphi_{2}\right)$ which ensure the boundedness of the commutator operators $\left[b, T_{\Omega}\right]$ from $L M_{p, \varphi_{1}}^{\left\{x_{0}\right\}}$ to $L M_{p, \varphi_{2}}^{\left\{x_{0}\right\}}, 1<p<\infty, \frac{1}{p}=\frac{1}{p_{1}}+\frac{1}{p_{2}}$.

By $A \lesssim B$ we mean that $A \leq C B$ with some positive constant $C$ independent of appropriate quantities. If $A \lesssim B$ and $B \lesssim A$, we write $A \approx B$ and say that $A$ and $B$ are equivalent.

\section{Generalized local Morrey spaces}

We find it convenient to define the generalized Morrey spaces in the form as follows.

Definition 2.1 Let $\varphi(x, r)$ be a positive measurable function on $\mathbb{R}^{n} \times(0, \infty)$ and $1 \leq p<$ $\infty$. We denote by $\mathcal{M}_{p, \varphi}\left(\mathbb{R}^{n}\right)$ the generalized Morrey space, the space of all functions $f \in$ $L_{p}^{\text {loc }}\left(\mathbb{R}^{n}\right)$ with finite quasinorm

$$
\|f\|_{\mathcal{M}_{p, \varphi}}=\sup _{x \in \mathbb{R}^{n}, r>0} \varphi(x, r)^{-1}\|f\|_{L_{p}(B(x, r))} .
$$

The generalized Morrey spaces $\mathcal{M}_{p, \varphi}\left(\mathbb{R}^{n}\right)$ with norm (2.1) introduced by Mizuhara in [12], which was later extended and studied by many authors (see [13, 14]). Note that the generalized Morrey spaces $M_{p, \varphi}\left(\mathbb{R}^{n}\right)$ with normalized form

$$
\|f\|_{M_{p, \varphi}}=\sup _{x \in \mathbb{R}^{n}, r>0} \varphi(x, r)^{-1}|B(x, r)|^{-\frac{1}{p}}\|f\|_{L_{p}(B(x, r))}
$$

were first defined by Guliyev in [15].

Also, in [15], there was defined the weak generalized Morrey space $W M_{p, \varphi} \equiv W M_{p, \varphi}\left(\mathbb{R}^{n}\right)$ of all functions $f \in W L_{p}^{\text {loc }}\left(\mathbb{R}^{n}\right)$ for which

$$
\|f\|_{W M_{p, \varphi}}=\sup _{x \in \mathbb{R}^{n}, r>0} \varphi(x, r)^{-1}|B(x, r)|^{-\frac{1}{p}}\|f\|_{W L_{p}(B(x, r))}<\infty .
$$

According to this definition, we recover the Morrey space $M_{p, \lambda}$ and weak Morrey space $W M_{p, \lambda}$ under the choice $\varphi(x, r)=r^{\frac{\lambda-n}{p}}$ :

$$
M_{p, \lambda}=\left.M_{p, \varphi}\right|_{\varphi(x, r)=r} \frac{\lambda-n}{p}, \quad W M_{p, \lambda}=\left.W M_{p, \varphi}\right|_{\varphi(x, r)=r} \frac{\lambda-n}{p} .
$$

Recall that in 1994 the doctoral thesis [16] by Guliyev (see also [17-20]) introduced the local Morrey-type space $L M_{p \theta, w}$ given by

$$
\|f\|_{L M_{p \theta, w}}=\|w(r)\| f\left\|_{L_{p}(B(0, r))}\right\|_{L_{\theta}(0, \infty)}<\infty
$$

where $w$ is a positive measurable function defined on $(0, \infty)$. The main purpose of [16] (also of [17-20]) is to give some sufficient conditions for the boundedness of fractional integral operators and singular integral operators defined on homogeneous Lie groups in the local Morrey-type space $L M_{p \theta, w}$. In a series of papers by Burenkov, $\mathrm{H}$ Guliyev and 
V Guliyev, etc. (see [21-24]), some necessary and sufficient conditions for the boundedness of fractional maximal operators, fractional integral operators, and singular integral operators in local Morrey-type spaces $L M_{p \theta, w}$ were given.

Definition 2.2 Let $\varphi(x, r)$ be a positive measurable function on $\mathbb{R}^{n} \times(0, \infty)$ and $1 \leq p<\infty$. We denote by $L M_{p, \varphi} \equiv L M_{p, \varphi}\left(\mathbb{R}^{n}\right)$ the generalized central (local) Morrey space, the space of all functions $f \in L_{p}^{\mathrm{loc}}\left(\mathbb{R}^{n}\right)$ with finite quasinorm

$$
\|f\|_{L M_{p, \varphi}}=\sup _{r>0} \varphi(0, r)^{-1}|B(0, r)|^{-\frac{1}{p}}\|f\|_{L_{p}(B(0, r))}
$$

Also by $W L M_{p, \varphi} \equiv W L M_{p, \varphi}\left(\mathbb{R}^{n}\right)$ we denote the weak generalized Morrey space of all functions $f \in W L_{p}^{\text {loc }}\left(\mathbb{R}^{n}\right)$ for which

$$
\|f\|_{W L M_{p, \varphi}}=\sup _{r>0} \varphi(0, r)^{-1}|B(0, r)|^{-\frac{1}{p}}\|f\|_{W L_{p}(B(0, r))}<\infty .
$$

Particularly, if $\theta=\infty, L M_{p \infty, w}=L M_{p, w}$, then the generalized central Morrey spaces $L M_{p, \varphi}$ are the same spaces as the local Morrey spaces $L M_{p \theta, w}$ with $w(r)=\varphi(0, r)^{-1} r^{-n / p}$. Note that $f \in M_{p, \varphi}$ if and only if $f(\cdot-x)_{x \in \mathbb{R}^{n}}$ forms a bounded set in $L M_{p \varphi}$.

Definition 2.3 Let $\varphi(x, r)$ be a positive measurable function on $\mathbb{R}^{n} \times(0, \infty)$ and $1 \leq p<\infty$. For any fixed $x_{0} \in \mathbb{R}^{n}$ we denote by $L M_{p, \varphi}^{\left\{x_{0}\right\}} \equiv L M_{p, \varphi}^{\left\{x_{0}\right\}}\left(\mathbb{R}^{n}\right)$ the generalized local Morrey space, the space of all functions $f \in L_{p}^{\operatorname{loc}}\left(\mathbb{R}^{n}\right)$ with finite quasinorm

$$
\|f\|_{L M_{p, \varphi}^{\left\{x_{0}\right\}}}=\left\|f\left(x_{0}+\cdot\right)\right\|_{L M_{p, \varphi}} .
$$

Also by $W L M_{p, \varphi}^{\left\{x_{0}\right\}} \equiv W L M_{p, \varphi}^{\left\{x_{0}\right\}}\left(\mathbb{R}^{n}\right)$ we denote the weak generalized local Morrey space of all functions $f \in W L_{p}^{\text {loc }}\left(\mathbb{R}^{n}\right)$ for which

$$
\|f\|_{W L M_{p, \varphi}^{\left\{x_{0}\right\}}}=\left\|f\left(x_{0}+\cdot\right)\right\|_{W L M_{p, \varphi}}<\infty
$$

According to this definition, we recover the local Morrey space $L M_{p, \lambda}^{\left\{x_{0}\right\}}$ and weak local Morrey space $W L M_{p, \lambda}^{\left\{x_{0}\right\}}$ under the choice $\varphi\left(x_{0}, r\right)=r^{\frac{\lambda-n}{p}}$ :

$$
L M_{p, \lambda}^{\left\{x_{0}\right\}}=\left.L M_{p, \varphi}^{\left\{x_{0}\right\}}\right|_{\varphi\left(x_{0}, r\right)=r} \frac{\lambda-n}{p}, \quad W L M_{p, \lambda}^{\left\{x_{0}\right\}}=\left.W L M_{p, \varphi}^{\left\{x_{0}\right\}}\right|_{\varphi\left(x_{0}, r\right)=r} \frac{\lambda-n}{p} .
$$

Wiener $[25,26]$ looked for a way to describe the behavior of a function at the infinity. The conditions he considered are related to appropriate weighted $L_{q}$ spaces. Beurling [27] extended this idea and defined a pair of dual Banach spaces $A_{q}$ and $B_{q^{\prime}}$, where $1 / q+1 / q^{\prime}=1$. To be precise, $A_{q}$ is a Banach algebra with respect to the convolution, expressed as a union of certain weighted $L_{q}$ spaces; the space $B_{q^{\prime}}$ is expressed as the intersection of the corresponding weighted $L_{q^{\prime}}$ spaces. Feichtinger [28] observed that the space $B_{q}$ can be described by

$$
\|f\|_{B_{q}}=\sup _{k \geq 0} 2^{-\frac{k n}{q}}\left\|f \chi_{k}\right\|_{L_{q}\left(\mathbb{R}^{n)}\right.},
$$


where $\chi_{0}$ is the characteristic function of the unit ball $\left\{x \in \mathbb{R}^{n}:|x| \leq 1\right\}, \chi_{k}$ is the characteristic function of the annulus $\left\{x \in \mathbb{R}^{n}: 2^{k-1}<|x| \leq 2^{k}\right\}, k=1,2, \ldots$. By duality, the space $A_{q}\left(\mathbb{R}^{n}\right)$, called the Beurling algebra now, can be described by

$$
\|f\|_{A_{q}}=\sum_{k=0}^{\infty} 2^{-\frac{k n}{q^{\prime}}}\left\|f \chi_{k}\right\|_{L_{q}\left(\mathbb{R}^{n}\right)} .
$$

Let $\dot{B}_{q}\left(\mathbb{R}^{n}\right)$ and $\dot{A}_{q}\left(\mathbb{R}^{n}\right)$ be the homogeneous versions of $B_{q}\left(\mathbb{R}^{n}\right)$ and $A_{q}\left(\mathbb{R}^{n}\right)$ by taking $k \in \mathbb{Z}$ in (2.3) and (2.4) instead of $k \geq 0$ there.

If $\lambda<0$ or $\lambda>n$, then $L M_{p, \lambda}^{\left\{x_{0}\right\}}\left(\mathbb{R}^{n}\right)=\Theta$, where $\Theta$ is the set of all functions equivalent to 0 on $\mathbb{R}^{n}$. Note that $L M_{p, 0}\left(\mathbb{R}^{n}\right)=L_{p}\left(\mathbb{R}^{n}\right)$ and $L M_{p, n}\left(\mathbb{R}^{n}\right)=\dot{B}_{p}\left(\mathbb{R}^{n}\right)$;

$$
\dot{B}_{p, \mu}=\left.L M_{p, \varphi}\right|_{\varphi(0, r)=r \mu n}, \quad W \dot{B}_{p, \mu}=\left.W L M_{p, \varphi}\right|_{\varphi(0, r)=r^{\mu n}} .
$$

Alvarez et al. [29], in order to study the relationship between central BMO spaces and Morrey spaces, introduced $\lambda$-central bounded mean oscillation spaces and central Morrey spaces $\dot{B}_{p, \mu}\left(\mathbb{R}^{n}\right) \equiv L M_{p, n+n p \mu}\left(\mathbb{R}^{n}\right), \mu \in\left[-\frac{1}{p}, 0\right]$. If $\mu<-\frac{1}{p}$ or $\mu>0$, then $\dot{B}_{p, \mu}\left(\mathbb{R}^{n}\right)=\Theta$. Note that $\dot{B}_{p,-\frac{1}{p}}\left(\mathbb{R}^{n}\right)=L_{p}\left(\mathbb{R}^{n}\right)$ and $\dot{B}_{p, 0}\left(\mathbb{R}^{n}\right)=\dot{B}_{p}\left(\mathbb{R}^{n}\right)$. Also define the weak central Morrey spaces $W \dot{B}_{p, \mu}\left(\mathbb{R}^{n}\right) \equiv W L M_{p, n+n p \mu}\left(\mathbb{R}^{n}\right)$.

Inspired by this, we consider the boundedness of singular integral operator with rough kernel on generalized local Morrey spaces and give the central bounded mean oscillation estimates for their commutators.

\section{Sublinear operators with rough kernel generated by Calderón-Zygmund operators in the spaces $L M_{p, \varphi}^{\left\{x_{0}\right\}}$}

In this section we are going to use the following statement on the boundedness of the weighted Hardy operator:

$$
H_{w} g(t):=\int_{t}^{\infty} g(s) w(s) d s, \quad 0<t<\infty,
$$

where $w$ is a fixed function non-negative and measurable on $(0, \infty)$.

The following theorem was proved in [30,31].

Theorem 3.1 Let $v_{1}, v_{2}$, and $w$ be positive almost everywhere and measurable functions on $(0, \infty)$. The inequality

$$
\underset{t>0}{\operatorname{ess} \sup } v_{2}(t) H_{w} g(t) \leq C \underset{t>0}{\operatorname{ess} \sup } v_{1}(t) g(t)
$$

holds for some $C>0$ for all non-negative and non-decreasing $g$ on $(0, \infty)$ if and only if

$$
B:=\underset{t>0}{\operatorname{ess} \sup } v_{2}(t) \int_{t}^{\infty} \frac{w(s) d s}{\operatorname{ess} \sup _{s<\tau<\infty} v_{1}(\tau)}<\infty .
$$

Moreover, the value $C=B$ is the best constant for (3.1).

The following statement, containing the results obtained in $[12,13]$ was proved in [3]. 
Theorem 3.2 Suppose that $\Omega \in L_{s}\left(S^{n-1}\right), s>1$, is homogeneous of degree zero and has mean value zero on $S^{n-1}$. Let $1 \leq s^{\prime}<p<\infty$ and $\varphi(x, r)$ satisfy the conditions

$$
c^{-1} \varphi(x, r) \leq \varphi(x, t) \leq c \varphi(x, r)
$$

whenever $r \leq t \leq 2 r$, where $c(\geq 1)$ does not depend on $t, r, x \in \mathbb{R}^{n}$, and

$$
\int_{r}^{\infty} \varphi(x, t)^{p} \frac{d t}{t} \leq C \varphi(x, r)^{p}
$$

where $C$ does not depend on $x$ and $r$. Then the operator $\bar{T}_{\Omega}$ is bounded on $M_{p, \varphi}$.

The following statement, containing the results obtained in $[12,13]$ was proved in $[15$, 16] (see also [17, 21-23, 32]).

Theorem 3.3 Let $1 \leq p<\infty$ and $\left(\varphi_{1}, \varphi_{2}\right)$ satisfy the condition

$$
\int_{r}^{\infty} \varphi_{1}(0, t) \frac{d t}{t} \leq C \varphi_{2}(0, r)
$$

where $C$ does not depend on $r$. Then the operator $\bar{T}$ is bounded from $L M_{p, \varphi_{1}}$ to $L M_{p, \varphi_{2}}$ for $p>1$ and from $L M_{1, \varphi_{1}}$ to $W L M_{1, \varphi_{2}}$ for $p=1$.

Corollary 3.4 Let $1 \leq p<\infty$ and $\left(\varphi_{1}, \varphi_{2}\right)$ satisfy the condition

$$
\int_{r}^{\infty} \varphi_{1}(x, t) \frac{d t}{t} \leq C \varphi_{2}(x, r)
$$

where $C$ does not depend on $x$ and $r$. Then the operator $\bar{T}$ is bounded from $M_{p, \varphi_{1}}$ to $M_{p, \varphi_{2}}$ for $p>1$ and from $M_{1, \varphi_{1}}$ to $W M_{1, \varphi_{2}}$ for $p=1$.

The following statement, containing results obtained in $[15,16]$, was proved in [30].

Theorem 3.5 Let $x_{0} \in \mathbb{R}^{n}, 1 \leq p<\infty$, and $\Omega \in L_{s}\left(S^{n-1}\right)$, $s>1$, be a homogeneous of degree zero. Let also, for $s^{\prime} \leq p$ or $p<s$, the pair $\left(\varphi_{1}, \varphi_{2}\right)$ satisfy the condition

$$
\int_{r}^{\infty} \frac{\operatorname{essinf}_{t<\tau<\infty} \varphi_{1}\left(x_{0}, \tau\right) \tau^{\frac{n}{p}}}{t^{\frac{n}{p}+1}} d t \leq C \varphi_{2}\left(x_{0}, r\right)
$$

where $C$ does not depend on $r$. Then the operator $\bar{T}_{\Omega}$ is bounded from $L M_{p, \varphi_{1}}^{\left\{x_{0}\right\}}$ to $L M_{p, \varphi_{2}}^{\left\{x_{0}\right\}}$ for $p>1$ and from $L M_{1, \varphi_{1}}^{\left\{x_{0}\right\}}$ to $W L M_{1, \varphi_{2}}^{\left\{x_{0}\right\}}$ for $p=1$.

Corollary 3.6 Let $1 \leq p<\infty, \Omega \in L_{s}\left(S^{n-1}\right), s>1$, be a homogeneous of degree zero. Let also, for $s^{\prime} \leq p$ or $p<s$, the pair $\left(\varphi_{1}, \varphi_{2}\right)$ satisfy the condition

$$
\int_{r}^{\infty} \frac{\operatorname{essinf}_{t<\tau<\infty} \varphi_{1}(x, \tau) \tau^{\frac{n}{p}}}{t^{\frac{n}{p}+1}} d t \leq C \varphi_{2}(x, r),
$$

where $C$ does not depend on $x$ and $r$. Then the operator $\bar{T}_{\Omega}$ is bounded from $M_{p, \varphi_{1}}$ to $M_{p, \varphi_{2}}$ for $p>1$ and from $M_{1, \varphi_{1}}$ to $W M_{1, \varphi_{2}}$ for $p=1$. 
Lemma 3.7 Let $x_{0} \in \mathbb{R}^{n}, 1 \leq p<\infty, T_{\Omega}$ be a sublinear operator satisfying condition (1.1) with $\Omega \in L_{s}\left(S^{n-1}\right), s>1$, be a homogeneous of degree zero, bounded on $L_{p}\left(\mathbb{R}^{n}\right)$ for $p>1$, and bounded from $L_{1}\left(\mathbb{R}^{n}\right)$ to $W L_{1}\left(\mathbb{R}^{n}\right)$.

If $p>1$ and $s^{\prime} \leq p$, then the inequality

$$
\left\|T_{\Omega} f\right\|_{L_{p}\left(B\left(x_{0}, r\right)\right)} \lesssim r^{\frac{n}{p}} \int_{2 r}^{\infty}\|f\|_{L_{p}\left(B\left(x_{0}, t\right)\right)} t^{-\frac{n}{p}-1} d t
$$

holds for any ball $B\left(x_{0}, r\right)$ and for all $f \in L_{p}^{\text {loc }}\left(\mathbb{R}^{n}\right)$.

If $p>1$ and $p<s$, then the inequality

$$
\left\|T_{\Omega} f\right\|_{L_{p}\left(B\left(x_{0}, r\right)\right)} \lesssim r^{\frac{n}{p}-\frac{n}{s}} \int_{2 r}^{\infty}\|f\|_{L_{p}\left(B\left(x_{0}, t\right)\right)} t^{\frac{n}{s}-\frac{n}{p}-1} d t
$$

holds for any ball $B\left(x_{0}, r\right)$ and for all $f \in L_{p}^{\text {loc }}\left(\mathbb{R}^{n}\right)$.

Moreover, for $s>1$ the inequality

$$
\left\|T_{\Omega} f\right\|_{W L_{1}\left(B\left(x_{0}, r\right)\right)} \lesssim r^{n} \int_{2 r}^{\infty} t^{-n-1}\|f\|_{L_{1}\left(B\left(x_{0}, t\right)\right)} d t
$$

holds for any ball $B\left(x_{0}, r\right)$ and for all $f \in L_{1}^{\text {loc }}\left(\mathbb{R}^{n}\right)$.

Proof Let $1<p<\infty$ and $s^{\prime} \leq p$. Set $B=B\left(x_{0}, r\right)$ for the ball centered at $x_{0}$ and of radius $r$. We represent $f$ as

$$
f=f_{1}+f_{2}, \quad f_{1}(y)=f(y) \chi_{2 B}(y), \quad f_{2}(y)=f(y) \chi_{\mathrm{c}_{(2 B)}}(y), \quad r>0,
$$

and have

$$
\left\|T_{\Omega} f\right\|_{L(B)} \leq\left\|T_{\Omega} f_{1}\right\|_{L_{p}(B)}+\left\|T_{\Omega} f_{2}\right\|_{L_{p}(B)} .
$$

Since $f_{1} \in L_{p}\left(\mathbb{R}^{n}\right), T_{\Omega} f_{1} \in L_{p}\left(\mathbb{R}^{n}\right)$ and from the boundedness of $T_{\Omega}$ on $L_{p}\left(\mathbb{R}^{n}\right)$ it follows that

$$
\left\|T_{\Omega} f_{1}\right\|_{L_{p}(B)} \leq\left\|T_{\Omega} f_{1}\right\|_{L_{p}\left(\mathbb{R}^{n}\right)} \leq C\left\|f_{1}\right\|_{L_{p}\left(\mathbb{R}^{n}\right)}=C\|f\|_{L_{p}(2 B)},
$$

where constant $C>0$ is independent of $f$.

Note that

$$
\begin{aligned}
\|\Omega(x-\cdot)\|_{L_{s}\left(B\left(x_{0}, t\right)\right)} & =\left(\int_{B\left(x-x_{0}, t\right)}|\Omega(y)|^{s} d y\right)^{\frac{1}{s}} \\
& \leq\left(\int_{B\left(0, t+\left|x-x_{0}\right|\right)}|\Omega(y)|^{s} d y\right)^{\frac{1}{s}} \\
& =\left(\int_{0}^{t+\left|x-x_{0}\right|} r^{n-1} d r \int_{S^{n-1}}\left|\Omega\left(y^{\prime}\right)\right|^{s} d \sigma\left(y^{\prime}\right)\right)^{\frac{1}{s}} \\
& =c_{0}\|\Omega\|_{L_{s}\left(S^{n-1}\right)}\left|B\left(0, t+\left|x-x_{0}\right|\right)\right|^{\frac{1}{s}},
\end{aligned}
$$

where $c_{0}=\left(n v_{n}\right)^{-1 / s}$ and $v_{n}=|B(0,1)|$. 
It is clear that $x \in B, y \in{ }^{\mathrm{C}}(2 B)$ implies $\frac{1}{2}\left|x_{0}-y\right| \leq|x-y| \leq \frac{3}{2}\left|x_{0}-y\right|$. We get

$$
\left|T_{\Omega} f_{2}(x)\right| \leq 2^{n} c_{1} \int_{\mathrm{C}_{(2 B)}} \frac{|f(y)||\Omega(x-y)|}{\left|x_{0}-y\right|^{n}} d y .
$$

By the Fubini theorem we have

$$
\begin{aligned}
\int_{\mathrm{C}_{(2 B)}} \frac{|f(y)||\Omega(x-y)|}{\left|x_{0}-y\right|^{n}} d y & \approx \int_{\mathrm{C}_{(2 B)}}|f(y)||\Omega(x-y)| \int_{\left|x_{0}-y\right|}^{\infty} \frac{d t}{t^{n+1}} d y \\
& \approx \int_{2 r}^{\infty} \int_{2 r \leq\left|x_{0}-y\right| \leq t}|f(y)||\Omega(x-y)| d y \frac{d t}{t^{n+1}} \\
& \lesssim \int_{2 r}^{\infty} \int_{B\left(x_{0}, t\right)}|f(y)||\Omega(x-y)| d y \frac{d t}{t^{n+1}} .
\end{aligned}
$$

Applying the Hölder inequality, we get

$$
\begin{aligned}
\int_{\mathrm{C}_{(2 B)}} \frac{|f(y)||\Omega(x-y)|}{\left|x_{0}-y\right|^{n}} d y & \lesssim \int_{2 r}^{\infty}\|f\|_{L_{p}\left(B\left(x_{0}, t\right)\right)}\|\Omega(x-\cdot)\|_{L_{s}\left(B\left(x_{0}, t\right)\right)}\left|B\left(x_{0}, t\right)\right|^{1-\frac{1}{p}-\frac{1}{s}} \frac{d t}{t^{n+1}} \\
& \lesssim \int_{2 r}^{\infty}\|f\|_{L_{p}\left(B\left(x_{0}, t\right)\right)}\left|B\left(x_{0}, t+\left|x-x_{0}\right|\right)\right|^{\frac{1}{s}}\left|B\left(x_{0}, t\right)\right|^{1-\frac{1}{p}-\frac{1}{s}} \frac{d t}{t^{n+1}} \\
& \lesssim \int_{2 r}^{\infty}\|f\|_{L_{p}\left(B\left(x_{0}, t\right)\right)}\left|B\left(x_{0}, t\right)\right|^{1-\frac{1}{p}} \frac{d t}{t^{n+1}} \\
& \approx \int_{2 r}^{\infty}\|f\|_{L_{p}\left(B\left(x_{0}, t\right)\right)} \frac{d t}{t^{\frac{n}{p}+1}} .
\end{aligned}
$$

Moreover, for all $p \in[1, \infty)$, the inequality

$$
\left\|T_{\Omega} f_{2}\right\|_{L_{p}(B)} \lesssim r^{\frac{n}{p}} \int_{2 r}^{\infty}\|f\|_{L_{p}\left(B\left(x_{0}, t\right)\right)} \frac{d t}{t^{\frac{n}{p}+1}}
$$

is valid. Thus

$$
\left\|T_{\Omega} f\right\|_{L_{p}(B)} \lesssim\|f\|_{L_{p}(2 B)}+r^{\frac{n}{p}} \int_{2 r}^{\infty}\|f\|_{L_{p}\left(B\left(x_{0}, t\right)\right)} \frac{d t}{t^{\frac{n}{p}+1}} .
$$

On the other hand,

$$
\|f\|_{L_{p}(2 B)} \approx r^{\frac{n}{p}}\|f\|_{L_{p}(2 B)} \int_{2 r}^{\infty} \frac{d t}{t^{\frac{n}{p}+1}} \leq r^{\frac{n}{p}} \int_{2 r}^{\infty}\|f\|_{L_{p}\left(B\left(x_{0}, t\right)\right)} \frac{d t}{t^{\frac{n}{p}+1}} .
$$

Thus

$$
\left\|T_{\Omega} f\right\|_{L_{p}(B)} \lesssim r^{\frac{n}{p}} \int_{2 r}^{\infty}\|f\|_{L_{p}\left(B\left(x_{0}, t\right)\right)} \frac{d t}{t^{\frac{n}{p}+1}} .
$$

When $1<p<s$, by the Fubini theorem, the Minkowski inequality and (3.10), we get

$$
\begin{aligned}
\left\|T_{\Omega} f_{2}\right\|_{L_{p}(B)} & \leq\left(\int_{B}\left(\int_{2 r}^{\infty} \int_{B\left(x_{0}, t\right)}|f(y)||\Omega(x-y)| d y \frac{d t}{t^{n+1}}\right)^{p} d x\right)^{\frac{1}{p}} \\
& \leq \int_{2 r}^{\infty} \int_{B\left(x_{0}, t\right)}|f(y)|\|\Omega(\cdot-y)\|_{L_{p}(B)} d y \frac{d t}{t^{n+1}}
\end{aligned}
$$




$$
\begin{aligned}
& \leq\left|B\left(x_{0}, r\right)\right|^{\frac{1}{p}-\frac{1}{s}} \int_{2 r}^{\infty} \int_{B\left(x_{0}, t\right)}|f(y)|\|\Omega(\cdot-y)\|_{L_{s}(B)} d y \frac{d t}{t^{n+1}} \\
& \leq\left|B\left(x_{0}, r\right)\right|^{\frac{1}{p}-\frac{1}{s}} \int_{2 r}^{\infty} \int_{B\left(x_{0}, t\right)}|f(y)|\left|B\left(x_{0}, r+\left|x_{0}-y\right|\right)\right|^{\frac{1}{s}} d y \frac{d t}{t^{n+1}} \\
& \lesssim r^{\frac{n}{p}-\frac{n}{s}} \int_{2 r}^{\infty}\|f\|_{L_{1}\left(B\left(x_{0}, t\right)\right)}\left|B\left(x_{0}, t\right)\right|^{\frac{1}{s}} \frac{d t}{t^{n+1}} \\
& \approx r^{\frac{n}{p}-\frac{n}{s}} \int_{2 r}^{\infty}\|f\|_{L_{p}\left(B\left(x_{0}, t\right) t^{\frac{n}{s}-\frac{n}{p}-1} d t .\right.}
\end{aligned}
$$

Let $p=1<s \leq \infty$. From the weak $(1,1)$ boundedness of $T_{\Omega}$ and (3.13) it follows that

$$
\begin{aligned}
\left\|T_{\Omega} f_{1}\right\|_{W L_{1}(B)} & \leq\left\|T_{\Omega} f_{1}\right\|_{W L_{1}\left(\mathbb{R}^{n}\right)} \lesssim\left\|f_{1}\right\|_{L_{1}\left(\mathbb{R}^{n}\right)} \\
& =\|f\|_{L_{1}(2 B)} \lesssim r^{n} \int_{2 r}^{\infty}\|f\|_{L_{1}\left(B\left(x_{0}, t\right)\right)} \frac{d t}{t^{n+1}} .
\end{aligned}
$$

Then from (3.12) and (3.15) we get the inequality (3.8).

Theorem 3.8 Let $x_{0} \in \mathbb{R}^{n}, 1 \leq p<\infty, T_{\Omega}$ be a sublinear operator satisfying condition (1.1) with $\Omega \in L_{s}\left(S^{n-1}\right), s>1$, be a homogeneous of degree zero. Suppose that the operator $T_{\Omega}$ is bounded on $L_{p}\left(\mathbb{R}^{n}\right)$ for $p>1$ and bounded from $L_{1}\left(\mathbb{R}^{n}\right)$ to $W L_{1}\left(\mathbb{R}^{n}\right)$. Let also, for $s^{\prime} \leq p$, $p \neq 1$, the pair $\left(\varphi_{1}, \varphi_{2}\right)$ satisfy the condition

$$
\int_{r}^{\infty} \frac{\operatorname{essinf}_{t<\tau<\infty} \varphi_{1}\left(x_{0}, \tau\right) \tau^{\frac{n}{p}}}{t^{\frac{n}{p}+1}} d t \leq C \varphi_{2}\left(x_{0}, r\right)
$$

and for $1<p<$ s the pair $\left(\varphi_{1}, \varphi_{2}\right)$ satisfy the condition

$$
\int_{r}^{\infty} \frac{\operatorname{essinf}_{t<\tau<\infty} \varphi_{1}\left(x_{0}, \tau\right) \tau^{\frac{n}{p}}}{t^{\frac{n}{p}-\frac{n}{s}+1}} d t \leq C \varphi_{2}\left(x_{0}, r\right) r^{\frac{n}{s}}
$$

where $C$ does not depend on $r$.

Then the operator $T_{\Omega}$ is bounded from $L M_{p, \varphi_{1}}^{\left\{x_{0}\right\}}$ to $L M_{p, \varphi_{2}}^{\left\{x_{0}\right\}}$. Moreover,

$$
\left\|T_{\Omega_{2}} f\right\|_{L M_{p, \varphi_{2}}^{\left\{x_{0}\right\}}} \lesssim\|f\|_{L M_{p, \varphi_{1}}^{\left\{x_{0}\right\}} \cdot}
$$

Also the operator $T_{\Omega}$ is bounded from $L M_{1, \varphi_{1}}^{\left\{x_{0}\right\}}$ to $W L M_{1, \varphi_{2}}^{\left\{x_{0}\right\}}$ and

$$
\left\|T_{\Omega} f\right\|_{W L M_{1, \varphi_{2}}^{\left\{x_{0}\right\}}} \lesssim\|f\|_{L M_{1, \varphi_{1}}^{\left\{x_{0}\right\}}}
$$

Proof Let $1<p<\infty$ and $s^{\prime} \leq p$. By Lemma 3.7 and Theorem 3.1 with $v_{2}(r)=\varphi_{2}\left(x_{0}, r\right)^{-1}$, $v_{1}(r)=\varphi_{1}\left(x_{0}, r\right)^{-1} r^{-\frac{n}{p}}, g(r)=\|f\|_{L_{p}\left(B\left(x_{0}, r\right)\right)}$, and $w(r)=r^{-\frac{n}{p}-1}$ we have

$$
\begin{aligned}
\left\|T_{\Omega} f\right\|_{L M_{p, \varphi_{2}}^{\left\{x_{0}\right\}}} & \lesssim \sup _{r>0} \varphi_{2}\left(x_{0}, r\right)^{-1} \int_{r}^{\infty}\|f\|_{L_{p}\left(B\left(x_{0}, t\right)\right)} \frac{d t}{t^{\frac{n}{p}+1}} \\
& \lesssim \sup _{r>0} \varphi_{1}\left(x_{0}, r\right)^{-1} r^{-\frac{n}{p}}\|f\|_{L_{p}\left(B\left(x_{0}, r\right)\right)}=\|f\|_{L M_{p, \varphi_{1}}^{\left\{x_{0}\right\}}} .
\end{aligned}
$$


Let $1<p<s$. By Lemma 3.7 and Theorem 3.1 with $v_{2}(r)=\varphi_{2}\left(x_{0}, r\right)^{-1}, v_{1}(r)=\varphi_{1}\left(x_{0}\right.$, $r)^{-1} r^{-\frac{n}{p}+\frac{n}{s}}, g(r)=\|f\|_{L_{p}\left(B\left(x_{0}, r\right)\right)}$, and $w(r)=r^{-\frac{n}{p}+\frac{n}{s}-1}$ we have

$$
\begin{aligned}
\left\|T_{\Omega} f\right\|_{L M_{p, \varphi_{2}}^{\left\{x_{0}\right\}}} & \lesssim \sup _{r>0} \varphi_{2}\left(x_{0}, r\right)^{-1} r^{-\frac{n}{s}} \int_{r}^{\infty}\|f\|_{L_{p}\left(B\left(x_{0}, t\right)\right)} \frac{d t}{t^{\frac{n}{p}-\frac{n}{s}+1}} \\
& \lesssim \sup _{r>0} \varphi_{1}\left(x_{0}, r\right)^{-1} r^{-\frac{n}{p}}\|f\|_{L_{p}\left(B\left(x_{0}, r\right)\right)}=\|f\|_{L M_{p, \varphi_{1}}^{\left\{x_{0}\right\}} .}
\end{aligned}
$$

Also for $p=1$

$$
\begin{aligned}
\left\|T_{\Omega} f\right\|_{W L M_{1, \varphi_{2}}^{\left\{x_{0}\right\}}} & \lesssim \sup _{r>0} \varphi_{2}\left(x_{0}, r\right)^{-1} \int_{r}^{\infty}\|f\|_{L_{1}\left(B\left(x_{0}, t\right)\right)} \frac{d t}{t^{n+1}} \\
& \lesssim \sup _{r>0} \varphi_{1}\left(x_{0}, r\right)^{-1} r^{-n}\|f\|_{L_{p}\left(B\left(x_{0}, r\right)\right)}=\|f\|_{L M_{1, \varphi_{1}}^{\left\{x_{0}\right\}} .}
\end{aligned}
$$

Corollary 3.9 Let $x_{0} \in \mathbb{R}^{n}, 1 \leq p<\infty$, $T_{\Omega}$ be a sublinear operator satisfying condition (1.1), with $\Omega \in L_{s}\left(S^{n-1}\right), s>1$, being a homogeneous of degree zero and bounded on $L_{p}\left(\mathbb{R}^{n}\right)$ for $p>1$, and bounded from $L_{1}\left(\mathbb{R}^{n}\right)$ to $W L_{1}\left(\mathbb{R}^{n}\right)$.

Let also, for $s^{\prime} \leq p, p \neq 1$, the pair $\left(\varphi_{1}, \varphi_{2}\right)$ satisfy the condition

$$
\int_{r}^{\infty} \frac{\operatorname{essinf}_{t<\tau<\infty} \varphi_{1}(x, \tau) \tau^{\frac{n}{p}}}{t^{\frac{n}{p}+1}} d t \leq C \varphi_{2}(x, r),
$$

and, for $1<p<s$, the pair $\left(\varphi_{1}, \varphi_{2}\right)$ satisfy the condition

$$
\int_{r}^{\infty} \frac{\operatorname{essinf}_{t<\tau<\infty} \varphi_{1}(x, \tau) \tau^{\frac{n}{p}}}{t^{\frac{n}{p}-\frac{n}{s}+1}} d t \leq C \varphi_{2}(x, r) r^{\frac{n}{s}},
$$

where $C$ does not depend on $x$ and $r$.

Then the operator $T_{\Omega}$ is bounded from $M_{p, \varphi_{1}}$ to $M_{p, \varphi_{2}}$. Moreover,

$$
\left\|T_{\Omega} f\right\|_{M_{p, \varphi_{2}}} \lesssim\|f\|_{M_{p, \varphi_{1}}}
$$

Also the operator $T_{\Omega}$ is bounded from $M_{1, \varphi_{1}}$ to $W M_{1, \varphi_{2}}$ and

$$
\left\|T_{\Omega} f\right\|_{W M_{1, \varphi_{2}}} \lesssim\|f\|_{M_{1, \varphi_{1}}} .
$$

Corollary 3.10 Let $1 \leq p<\infty$ and $\left(\varphi_{1}, \varphi_{2}\right)$ satisfy condition (3.16). Then the operator $\bar{T}$ is bounded from $L M_{p, \varphi_{1}}^{\left\{x_{0}\right\}}$ to $L M_{p, \varphi_{2}}^{\left\{x_{0}\right\}}$ for $p>1$ and from $L M_{1, \varphi_{1}}^{\left\{x_{0}\right\}}$ to $W L M_{1, \varphi_{2}}^{\left\{x_{0}\right\}}$.

Let $f \in L_{1}^{\text {loc }}\left(\mathbb{R}^{n}\right)$. The rough Hardy-Littlewood maximal function $M_{\Omega}$ is defined by

$$
M_{\Omega} f(x)=\sup _{t>0} \frac{1}{|B(x, t)|} \int_{B(x, t)}|\Omega(x-y)||f(y)| d y .
$$

Then we can give the following corollary.

Corollary 3.11 Let $1 \leq p<\infty, \Omega \in L_{s}\left(S^{n-1}\right)$. For $s^{\prime} \leq p, p \neq 1$, the pair $\left(\varphi_{1}, \varphi_{2}\right)$ satisfies condition (3.16) and, for $1<p<s$, the pair $\left(\varphi_{1}, \varphi_{2}\right)$ satisfies condition (3.17). Then the operators $M_{\Omega}$ and $\bar{T}_{\Omega}$ are bounded from $L M_{p, \varphi_{1}}^{\left\{x_{0}\right\}}$ to $L M_{p, \varphi_{2}}^{\left\{x_{0}\right\}}$, for $p>1$, and from $L M_{1, \varphi_{1}}^{\left\{x_{0}\right\}}$ to $W L M_{1, \varphi_{2}}^{\left\{x_{0}\right\}}$. 
Corollary 3.12 Let $1 \leq p<\infty, \Omega \in L_{s}\left(S^{n-1}\right)$. For $s^{\prime} \leq p, p \neq 1$, the pair $\left(\varphi_{1}, \varphi_{2}\right)$ satisfies condition (3.18) and, for $1<p<s$, the pair $\left(\varphi_{1}, \varphi_{2}\right)$ satisfies condition (3.19). Then the operators $M_{\Omega}$ and $\bar{T}_{\Omega}$ are bounded from $M_{p, \varphi_{1}}$ to $M_{p, \varphi_{2}}$ and from $M_{1, \varphi_{1}}$ to $W M_{1, \varphi_{2}}$.

Remark 3.13 Note that, in the case $s=\infty$, Corollary 3.9 was proved in [33]. The condition (3.16) in Theorem 3.8 is weaker than condition (3.4) in Theorem 3.3 (see [33]).

\section{Commutators of linear operators with rough kernel generated by Calderón-Zygmund operators in the spaces $L M_{p, \varphi}^{\left\{x_{0}\right\}}$}

Let $T$ be a linear operator; for a function $b$, we define the commutator $[b, T]$ by

$$
[b, T] f(x)=b(x) T f(x)-T(b f)(x)
$$

for any suitable function $f$. Let $\widetilde{T}$ be a Calderón-Zygmund singular integral operator. A well-known result of Coifman et al. [34] states that the commutator $[b, \widetilde{T}] f=b \widetilde{T} f-$ $\widetilde{T}(b f)$ is bounded on $L_{p}\left(\mathbb{R}^{n}\right), 1<p<\infty$, if and only if $b \in B M O\left(\mathbb{R}^{n}\right)$. The commutator of Calderón-Zygmund operators plays an important role in studying the regularity of solutions of elliptic partial differential equations of second order (see, for example, [8-10, 35]).

The definition of a local Campanato space is as follows.

Definition 4.1 Let $1 \leq q<\infty$ and $0 \leq \lambda<\frac{1}{n}$. A function $f \in L_{q}^{\text {loc }}\left(\mathbb{R}^{n}\right)$ is said to belong to the $L C_{q, \lambda}^{\left\{x_{0}\right\}}\left(\mathbb{R}^{n}\right)$ (local Campanato space), if

$$
\|f\|_{L C_{q, \lambda}^{\left\{x_{0}\right\}}}=\sup _{r>0}\left(\frac{1}{\left|B\left(x_{0}, r\right)\right|^{1+\lambda q}} \int_{B\left(x_{0}, r\right)}\left|f(y)-f_{B\left(x_{0}, r\right)}\right|^{q} d y\right)^{1 / q}<\infty
$$

where

$$
f_{B\left(x_{0}, r\right)}=\frac{1}{\left|B\left(x_{0}, r\right)\right|} \int_{B\left(x_{0}, r\right)} f(y) d y .
$$

Define

$$
L C_{q, \lambda}^{\left\{x_{0}\right\}}\left(\mathbb{R}^{n}\right)=\left\{f \in L_{q}^{\text {loc }}\left(\mathbb{R}^{n}\right):\|f\|_{\left.L C_{q, \lambda}^{\left\{x_{0}\right\}}<\infty\right\}}\right.
$$

In [36], Lu and Yang introduced the central BMO space $C B M O_{q}\left(\mathbb{R}^{n}\right)=L C_{q, 0}^{\{0\}}\left(\mathbb{R}^{n}\right)$. Note that $B M O\left(\mathbb{R}^{n}\right) \subset \bigcap_{q>1} C B M O_{q}^{\left\{x_{0}\right\}}\left(\mathbb{R}^{n}\right), 1 \leq q<\infty$. The space $C B M O_{q}^{\left\{x_{0}\right\}}\left(\mathbb{R}^{n}\right)$ can be regarded as a local version of $B M O\left(\mathbb{R}^{n}\right)$, the space of bounded mean oscillation, at the origin. But they have quite different properties. The classical John-Nirenberg inequality shows that functions in $B M O\left(\mathbb{R}^{n}\right)$ are locally exponentially integrable. This implies that, for any $1 \leq q<\infty$, the functions in $B M O\left(\mathbb{R}^{n}\right)$ can be described by means of the condition:

$$
\sup _{B \subset \mathbb{R}^{n}}\left(\frac{1}{|B|} \int_{B}\left|f(y)-f_{B}\right|^{q} d y\right)^{1 / q}<\infty
$$

where $B$ denotes an arbitrary ball in $\mathbb{R}^{n}$. However, the space $C B M O_{q}^{\left\{x_{0}\right\}}\left(\mathbb{R}^{n}\right)$ depends on $q$. If $q_{1}<q_{2}$, then $C B M O_{q_{2}}^{\left\{x_{0}\right\}}\left(\mathbb{R}^{n}\right) \varsubsetneqq C B M O_{q_{1}}^{\left\{x_{0}\right\}}\left(\mathbb{R}^{n}\right)$. Therefore, there is no analogy of the famous 
John-Nirenberg inequality of $B M O\left(\mathbb{R}^{n}\right)$ for the space $C B M O_{q}^{\left\{x_{0}\right\}}\left(\mathbb{R}^{n}\right)$. One can imagine that the behavior of $C B M O_{q}^{\left\{x_{0}\right\}}\left(\mathbb{R}^{n}\right)$ may be quite different from that of $B M O\left(\mathbb{R}^{n}\right)$.

We will use the following statement on the boundedness of the weighted Hardy operator:

$$
H_{w}^{*} g(r):=\int_{r}^{\infty}\left(1+\ln \frac{t}{r}\right) g(t) w(t) d t, \quad r \in(0, \infty)
$$

where $w$ is a weight.

The following theorem was proved in [37].

Theorem 4.2 ([37]) Let $v_{1}, v_{2}$, and $w$ be positive almost everywhere and measurable functions on $(0, \infty)$. The inequality

$$
\underset{r>0}{\operatorname{ess} \sup } v_{2}(r) H_{w}^{*} g(r) \leq \underset{r>0}{\operatorname{ess} \sup } v_{1}(r) g(r)
$$

holds, for some $C>0$ for all non-negative and non-decreasing $g$ on $(0, \infty)$, if and only if

$$
B:=\sup _{r>0} v_{2}(r) \int_{r}^{\infty}\left(1+\ln \frac{t}{r}\right) \frac{w(t) d t}{\sup _{t<s<\infty} v_{1}(s)}<\infty .
$$

Moreover, the value $C=B$ is the best constant for (4.1).

Remark 4.3 In (4.1)-(4.2) it is assumed that $0 \cdot \infty=0$.

Lemma 4.4 Let $b$ be a function in $L C_{q, \lambda}^{\left\{x_{0}\right\}}\left(\mathbb{R}^{n}\right), 1 \leq q<\infty, 0 \leq \lambda<\frac{1}{n}$, and $r_{1}, r_{2}>0$. Then

$$
\left(\frac{1}{\left|B\left(x_{0}, r_{1}\right)\right|^{1+\lambda q}} \int_{B\left(x_{0}, r_{1}\right)}\left|b(y)-b_{B\left(x_{0}, r_{2}\right)}\right|^{q} d y\right)^{\frac{1}{q}} \leq C\left(1+\left|\ln \frac{r_{1}}{r_{2}}\right|\right)\|b\|_{L C_{q, \lambda}^{\left\{x_{0}\right\}}},
$$

where $C>0$ is independent of $b, r_{1}$, and $r_{2}$.

In [3] the following statement was proved for the commutators of singular integral operators with rough kernels, containing the result in $[12,13]$.

Theorem 4.5 Suppose that $\Omega \in L_{s}\left(S^{n-1}\right), s>1$, is homogeneous of degree zero and $b \in$ $B M O\left(\mathbb{R}^{n}\right)$. Let $1 \leq s^{\prime}<p<\infty, \varphi(x, r)$ satisfy the conditions (3.2) and (3.3). Then the operator $\left[b, \bar{T}_{\Omega}\right]$ is bounded on $M_{p, \varphi}$.

Lemma 4.6 Let $x_{0} \in \mathbb{R}^{n}, 1<p<\infty, b \in L C_{p_{2}, \lambda}^{\left\{x_{0}\right\}}\left(\mathbb{R}^{n}\right), \frac{1}{p}=\frac{1}{p_{1}}+\frac{1}{p_{2}}$, and $0 \leq \lambda<\frac{1}{n}$. Let also $T_{\Omega}$ be a linear operator satisfying condition (1.1) with $\Omega \in L_{s}\left(S^{n-1}\right), s>1$, be a homogeneous of degree zero and bounded on $L_{p}\left(\mathbb{R}^{n}\right)$ for $1<p<\infty$.

Then, for $s^{\prime} \leq p_{1}$, the inequality

$$
\left\|\left[b, T_{\Omega}\right] f\right\|_{L_{p}\left(B\left(x_{0}, r\right)\right)} \lesssim\|b\|_{L C_{p_{2}, \lambda}^{\left(x_{0}\right]}} r^{\frac{n}{p}} \int_{2 r}^{\infty}\left(1+\ln \frac{t}{r}\right) t^{n \lambda-\frac{n}{p_{1}}-1}\|f\|_{L_{p_{1}}\left(B\left(x_{0}, t\right)\right)} d t
$$

holds, for any ball $B\left(x_{0}, r\right)$ and for all $f \in L_{p_{1}}^{\text {loc }}\left(\mathbb{R}^{n}\right)$. 
Also, for $p_{1}<s$, the inequality

$$
\left\|\left[b, T_{\Omega}\right] f\right\|_{L_{p}\left(B\left(x_{0}, r\right)\right)} \lesssim\|b\|_{L C_{p_{2}, \lambda}^{\left(x_{0}\right)}} r^{\frac{n}{p}-\frac{n}{s}} \int_{2 r}^{\infty}\left(1+\ln \frac{t}{r}\right) t^{n \lambda-\frac{n}{p_{1}}+\frac{n}{s}-1}\|f\|_{L_{p_{1}}\left(B\left(x_{0}, t\right)\right)} d t
$$

holds, for any ball $B\left(x_{0}, r\right)$ and for all $f \in L_{p_{1}}^{\mathrm{loc}}\left(\mathbb{R}^{n}\right)$.

Proof Let $1<p<\infty, b \in L C_{p_{2}, \lambda}^{\left\{x_{0}\right\}}\left(\mathbb{R}^{n}\right)$, and $\frac{1}{p}=\frac{1}{p_{1}}+\frac{1}{p_{2}}$. As in the proof of Lemma 3.7, we represent the function $f$ in the form (3.9) and have

$$
\begin{aligned}
{\left[b, T_{\Omega}\right] f(x) \equiv } & J_{1}+J_{2}+J_{3}+J_{4}=\left(b(x)-b_{B}\right) T_{\Omega} f_{1}(x) \\
& -T_{\Omega}\left(\left(b(\cdot)-b_{B}\right) f_{1}\right)(x)+\left(b(x)-b_{B}\right) T_{\Omega} f_{2}(x)-T_{\Omega}\left(\left(b(\cdot)-b_{B}\right) f_{2}\right)(x) .
\end{aligned}
$$

Hence we get

$$
\left\|\left[b, T_{\Omega}\right] f\right\|_{L_{p}(B)} \leq\left\|J_{1}\right\|_{L_{p}(B)}+\left\|J_{2}\right\|_{L_{p}(B)}+\left\|J_{3}\right\|_{L_{p}(B)}+\left\|J_{4}\right\|_{L_{p}(B)} .
$$

From the boundedness of $T_{\Omega}$ on $L_{p}\left(\mathbb{R}^{n}\right)$ and Lemma 4.4 it follows that

$$
\begin{aligned}
& \left\|J_{1}\right\|_{L_{p}(B)} \leq\left\|\left(b(\cdot)-b_{B}\right) T_{\Omega_{2}} f_{1}(\cdot)\right\|_{L_{p}\left(\mathbb{R}^{n}\right)} \\
& \leq\left\|\left(b(\cdot)-b_{B}\right)\right\|_{L_{p_{2}}\left(\mathbb{R}^{n}\right)}\left\|T_{\Omega_{2}} f_{1}(\cdot)\right\|_{L_{p_{1}}\left(\mathbb{R}^{n}\right)} \\
& \left.\leq C\|b\|_{L C_{p_{2}, \lambda}^{\left\{x_{0}\right\}}}\right\}^{\frac{n}{p_{2}}+n \lambda}\left\|f_{1}\right\|_{L_{p_{1}}\left(\mathbb{R}^{n}\right)} \\
& =C\|b\|_{L C_{p_{2}, \lambda}^{\left\{x_{0}\right\}}} r^{\frac{n}{p_{2}}+\frac{n}{p_{1}}+n \lambda}\|f\|_{L_{p_{1}}(2 B)} \int_{2 r}^{\infty} t^{-1-\frac{n}{p_{1}}} d t \\
& \lesssim\|b\|_{\left.L C_{p_{2}, \lambda}^{\left\{x_{0}\right\}}\right\}} r_{2 r}^{\frac{n}{p}} \int^{\infty}\left(1+\ln \frac{t}{r}\right) t^{n \lambda-\frac{n}{p_{1}}-1}\|f\|_{L_{p_{1}}\left(B\left(x_{0}, t\right)\right)} d t .
\end{aligned}
$$

From Lemma 4.4 for $J_{2}$ we have

$$
\begin{aligned}
& \left\|J_{2}\right\|_{L_{p}(B)} \leq\left\|T_{\Omega}\left(b(\cdot)-b_{B}\right) f_{1}\right\|_{L_{p}\left(\mathbb{R}^{n}\right)} \\
& \lesssim\left\|\left(b(\cdot)-b_{B}\right) f_{1}\right\|_{L_{p}\left(\mathbb{R}^{n}\right)} \\
& \lesssim\left\|b(\cdot)-b_{B}\right\|_{L_{p_{2}}\left(\mathbb{R}^{n}\right)}\left\|f_{1}\right\|_{L_{p_{1}}\left(\mathbb{R}^{n}\right)} \\
& \lesssim\|b\|_{L C_{p_{2}, \lambda}^{\left\{x_{0}, \lambda\right.}} r^{\frac{n}{p_{2}}+\frac{n}{p_{1}}+n \lambda}\|f\|_{L_{p_{1}}(2 B)} \int_{2 r}^{\infty} t^{-1-\frac{n}{p_{1}}} d t \\
& \lesssim\|b\|_{L C_{p_{2}, \lambda}^{\left[x_{0}\right\}}} r^{\frac{n}{p}} \int_{2 r}^{\infty}\left(1+\ln \frac{t}{r}\right) t^{n \lambda-\frac{n}{p_{1}}-1}\|f\|_{L_{p_{1}}\left(B\left(x_{0}, t\right)\right)} d t .
\end{aligned}
$$

For $J_{3}$, it is known that $x \in B, y \in{ }^{\mathrm{C}}(2 B)$, which implies $\frac{1}{2}\left|x_{0}-y\right| \leq|x-y| \leq \frac{3}{2}\left|x_{0}-y\right|$. When $s^{\prime} \leq p_{1}$, by the Fubini theorem and (3.10), and applying the Hölder inequality, we have

$$
\begin{aligned}
\left|T_{\Omega} f_{2}(x)\right| & \leq c_{0} \int_{\mathrm{C}_{(2 B)}}|\Omega(x-y)| \frac{|f(y)|}{\left|x_{0}-y\right|^{n}} d y \\
& \approx \int_{2 r}^{\infty} \int_{2 r<\left|x_{0}-y\right|<t}|\Omega(x-y)||f(y)| d y t^{-1-n} d t
\end{aligned}
$$




$$
\begin{aligned}
& \lesssim \int_{2 r}^{\infty} \int_{B\left(x_{0}, t\right)}|\Omega(x-y)||f(y)| d y t^{-1-n} d t \\
& \lesssim \int_{2 r}^{\infty}\|f\|_{L_{p_{1}}\left(B\left(x_{0}, t\right)\right)}\|\Omega(x-\cdot)\|_{L_{S}\left(B\left(x_{0}, t\right)\right)}\left|B\left(x_{0}, t\right)\right|^{1-\frac{1}{p_{1}}-\frac{1}{s}} t^{-1-n} d t \\
& \lesssim \int_{2 r}^{\infty}\|f\|_{L_{p_{1}}\left(B\left(x_{0}, t\right)\right)}\left|B\left(x_{0}, t+\left|x-x_{0}\right|\right)\right|^{\frac{1}{s}}\left|B\left(x_{0}, t\right)\right|^{1-\frac{1}{p_{1}}-\frac{1}{s}} \frac{d t}{t^{n+1}} \\
& \lesssim \int_{2 r}^{\infty} t^{-\frac{n}{p_{1}}-1}\|f\|_{L_{p_{1}}\left(B\left(x_{0}, t\right)\right)} d t .
\end{aligned}
$$

Hence, from Lemma 4.4 we get

$$
\begin{aligned}
\left\|J_{3}\right\|_{L_{p}(B)} & =\left\|\left(b(\cdot)-b_{B}\right) T_{\Omega} f_{2}(\cdot)\right\|_{L_{p}\left(\mathbb{R}^{n}\right)} \\
& \leq\left\|\left(b(\cdot)-b_{B}\right)\right\|_{L_{p}\left(\mathbb{R}^{n}\right)} \int_{2 r}^{\infty} t^{-\frac{n}{p_{1}}-1}\|f\|_{L_{p_{1}}\left(B\left(x_{0}, t\right)\right)} d t \\
& \leq\left\|\left(b(\cdot)-b_{B}\right)\right\|_{L_{p_{2}}\left(\mathbb{R}^{n}\right)^{n}} r^{\frac{n}{p_{1}}} \int_{2 r}^{\infty} t^{-\frac{n}{p_{1}}-1}\|f\|_{L_{p_{1}}\left(B\left(x_{0}, t\right)\right)} d t \\
& \lesssim\|b\|_{L C_{p_{2}, \lambda}^{\left[x_{0}\right\}}} r^{\frac{n}{p}+n \lambda} \int_{2 r}^{\infty}\left(1+\ln \frac{t}{r}\right) t^{-\frac{n}{p_{1}}-1}\|f\|_{L_{p_{1}}\left(B\left(x_{0}, t\right)\right)} d t \\
& \lesssim\|b\|_{L C_{p_{2}, \lambda}^{\left[x_{0}\right)} r^{\frac{n}{p}}} \int_{2 r}^{\infty}\left(1+\ln \frac{t}{r}\right) t^{n \lambda-\frac{n}{p_{1}}-1}\|f\|_{L_{p_{1}}\left(B\left(x_{0}, t\right)\right)} d t .
\end{aligned}
$$

When $p_{1}<s$, by the Fubini theorem, the Minkowski inequality, (3.10) and from Lemma 4.4, we get

$$
\begin{aligned}
& \left\|J_{3}\right\|_{L_{p}(B)} \leq\left(\int_{B}\left(\int_{2 r}^{\infty} \int_{B\left(x_{0}, t\right)}|f(y)|\left|b(x)-b_{B}\right||\Omega(x-y)| d y \frac{d t}{t^{n+1}}\right)^{p} d x\right)^{\frac{1}{p}} \\
& \leq \int_{2 r}^{\infty} \int_{B\left(x_{0}, t\right)}|f(y)|\left\|\left(b(\cdot)-b_{B}\right) \Omega(\cdot-y)\right\|_{L_{p}(B)} d y \frac{d t}{t^{n+1}} \\
& \leq \int_{2 r}^{\infty} \int_{B\left(x_{0}, t\right)}|f(y)|\left\|b(\cdot)-b_{B}\right\|_{L_{p_{2}}(B)}\|\Omega(\cdot-y)\|_{L_{p_{1}}(B)} d y \frac{d t}{t^{n+1}} \\
& \lesssim\|b\|_{L C_{p_{2}, \lambda}^{\left(x_{0}, \lambda\right.}} r^{\frac{n}{p_{2}}+n \lambda}|B|^{\frac{1}{p_{1}}-\frac{1}{s}} \int_{2 r}^{\infty} \int_{B\left(x_{0}, t\right)}|f(y)|\|\Omega(\cdot-y)\|_{L_{s}(B)} d y \frac{d t}{t^{n+1}} \\
& \lesssim\|b\|_{L C_{p_{2}, \lambda}^{\left\{x_{0}\right\}}} r^{\frac{n}{p}-\frac{n}{s}+n \lambda} \int_{2 r}^{\infty}\|f\|_{L_{1}\left(B\left(x_{0}, t\right)\right)}\left|B\left(x_{0}, t+\left|x_{0}-y\right|\right)\right|^{\frac{1}{s}} \frac{d t}{t^{n+1}} \\
& \lesssim\|b\|_{L C_{p_{2}, \lambda}^{\left\{x_{0}, \lambda\right.}} r^{\frac{n}{p}-\frac{n}{s}} \int_{2 r}^{\infty}\left(1+\ln \frac{t}{r}\right) t^{n \lambda+\frac{n}{s}-\frac{n}{p_{1}}-1}\|f\|_{L_{p_{1}}\left(B\left(x_{0}, t\right)\right)} d t .
\end{aligned}
$$

For $x \in B$, by the Fubini theorem, applying the Hölder inequality, and from Lemma 4.4 we have

$$
\begin{aligned}
& \left|T_{\Omega}\left(\left(b(\cdot)-b_{B}\right) f_{2}\right)(x)\right| \\
& \quad \lesssim \int_{\mathrm{C}_{(2 B)}}\left|b(y)-b_{B}\right||\Omega(x-y)| \frac{|f(y)|}{|x-y|^{n}} d y \\
& \quad \lesssim \int_{\mathrm{C}_{(2 B)}}\left|b(y)-b_{B}\right||\Omega(x-y)| \frac{|f(y)|}{\left|x_{0}-y\right|^{n}} d y
\end{aligned}
$$




$$
\begin{aligned}
& \approx \int_{2 r}^{\infty} \int_{2 r<\left|x_{0}-y\right|<t}\left|b(y)-b_{B}\right||\Omega(x-y)||f(y)| d y \frac{d t}{t^{n+1}} \\
& \lesssim \int_{2 r}^{\infty} \int_{B\left(x_{0}, t\right)}\left|b(y)-b_{B\left(x_{0}, t\right)}\right||\Omega(x-y)||f(y)| d y \frac{d t}{t^{n+1}} \\
& +\int_{2 r}^{\infty}\left|b_{B\left(x_{0}, r\right)}-b_{B\left(x_{0}, t\right)}\right| \int_{B\left(x_{0}, t\right)}|\Omega(x-y)||f(y)| d y \frac{d t}{t^{n+1}} \\
& \lesssim \int_{2 r}^{\infty}\left\|\left(b(\cdot)-b_{B\left(x_{0}, t\right)}\right) f\right\|_{L_{p}\left(B\left(x_{0}, t\right)\right)}\|\Omega(\cdot-y)\|_{L_{s}\left(B\left(x_{0}, t\right)\right)}\left|B\left(x_{0}, t\right)\right|^{1-\frac{1}{p}-\frac{1}{s}} \frac{d t}{t^{n+1}} \\
& +\int_{2 r}^{\infty}\left|b_{B\left(x_{0}, r\right)}-b_{B\left(x_{0}, t\right)}\right|\|f\|_{L_{p_{1}}\left(B\left(x_{0}, t\right)\right)}\|\Omega(\cdot-y)\|_{L_{s}\left(B\left(x_{0}, t\right)\right)}\left|B\left(x_{0}, t\right)\right|^{1-\frac{1}{p_{1}}-\frac{1}{s}} t^{-n-1} d t \\
& \lesssim \int_{2 r}^{\infty}\left\|b(\cdot)-b_{B\left(x_{0}, t\right)}\right\|_{L_{p_{2}}\left(B\left(x_{0}, t\right)\right)}\|f\|_{L_{p_{1}}\left(B\left(x_{0}, t\right)\right)} t^{-1-\frac{n}{p_{1}}} d t \\
& +\|b\|_{L C_{p_{2}, \lambda}^{\left\{x_{0}\right\}}} \int_{2 r}^{\infty}\left(1+\ln \frac{t}{r}\right) t^{n \lambda-\frac{n}{p_{1}}-1}\|f\|_{L_{p_{1}}\left(B\left(x_{0}, t\right)\right)} d t \\
& \lesssim\|b\|_{L C_{p_{2}, \lambda}^{\left\{x_{0}\right\}}} \int_{2 r}^{\infty}\left(1+\ln \frac{t}{r}\right) t^{n \lambda-\frac{n}{p_{1}}-1}\|f\|_{L_{p_{1}}\left(B\left(x_{0}, t\right)\right)} d t .
\end{aligned}
$$

Then for $J_{4}$ we have

$$
\begin{aligned}
\left\|J_{4}\right\|_{L_{p}(B)} & \leq\left\|T_{\Omega}\left(b(\cdot)-b_{B}\right) f_{2}\right\|_{L_{p}\left(\mathbb{R}^{n}\right)} \\
& \lesssim\|b\|_{L C_{p_{2}, \lambda}^{\left\{x_{0}\right\}}} r^{\frac{n}{p}} \int_{2 r}^{\infty}\left(1+\ln \frac{t}{r}\right) t^{n \lambda-\frac{n}{p_{1}}-1}\|f\|_{L_{p_{1}}\left(B\left(x_{0}, t\right)\right)} d t
\end{aligned}
$$

When $p_{1}<s$, by the Fubini theorem, (3.10), and the Minkowski inequality, we get

$$
\begin{aligned}
\left\|T_{\Omega} f_{2}\right\|_{L_{p}(B)} & \leq\left(\int_{B}\left(\int_{2 r}^{\infty} \int_{B\left(x_{0}, t\right)}|f(y)||\Omega(x-y)| d y \frac{d t}{t^{n+1}}\right)^{p} d x\right)^{\frac{1}{p}} \\
& \leq \int_{2 r}^{\infty} \int_{B\left(x_{0}, t\right)}|f(y)|\|\Omega(\cdot-y)\|_{L_{p}(B)} d y \frac{d t}{t^{n+1}} \\
& \leq|B|^{\frac{1}{p}-\frac{1}{s}} \int_{2 r}^{\infty} \int_{B\left(x_{0}, t\right)}|f(y)|\|\Omega(\cdot-y)\|_{L_{s}(B)} d y \frac{d t}{t^{n+1}} \\
& \lesssim r^{\frac{n}{p}-\frac{n}{s}} \int_{2 r}^{\infty}\|f\|_{L_{1}\left(B\left(x_{0}, t\right)\right)}\left|B\left(x_{0}, t+\left|x_{0}-y\right|\right)\right|^{\frac{1}{s}} \frac{d t}{t^{n+1}} \\
& \lesssim r^{\frac{n}{p}-\frac{n}{s}} \int_{2 r}^{\infty} t^{\frac{n}{s}-\frac{n}{p_{1}}-1}\|f\|_{L_{p_{1}}\left(B\left(x_{0}, t\right)\right)} d t .
\end{aligned}
$$

Now combining all the above estimates, we end the proof of Lemma 4.6.

The following theorem is true.

Theorem 4.7 Suppose that $x_{0} \in \mathbb{R}^{n}, 1<p<\infty, T_{\Omega}$ is a linear operator satisfying condition (1.1) with $\Omega \in L_{s}\left(S^{n-1}\right)$, $s>1$, is homogeneous of degree zero and bounded on $L_{p}\left(\mathbb{R}^{n}\right)$. Let $b \in L C_{p_{2}, \lambda}^{\left\{x_{0}\right\}}\left(\mathbb{R}^{n}\right), \frac{1}{p}=\frac{1}{p_{1}}+\frac{1}{p_{2}}, 0 \leq \lambda<\frac{1}{n}$.

Let also, for $s^{\prime} \leq p_{1}$, the pair $\left(\varphi_{1}, \varphi_{2}\right)$ satisfy the condition

$$
\int_{r}^{\infty}\left(1+\ln \frac{t}{r}\right) \frac{\operatorname{essinf}_{t<\tau<\infty} \varphi_{1}\left(x_{0}, \tau\right) \tau^{\frac{n}{p}}}{t^{\frac{n}{p}+1-n \lambda}} d t \leq C \varphi_{2}\left(x_{0}, r\right),
$$


and, for $p_{1}<s$, the pair $\left(\varphi_{1}, \varphi_{2}\right)$ satisfy the condition

$$
\int_{r}^{\infty}\left(1+\ln \frac{t}{r}\right) \frac{\operatorname{essinf}_{t<\tau<\infty} \varphi_{1}(x, \tau) \tau^{\frac{n}{p}}}{t^{\frac{n}{p}-\frac{n}{s}+1}} d t \leq C \varphi_{2}(x, r) r^{\frac{n}{s}}
$$

where $C$ does not depend on $r$.

Then the operator $\left[b, T_{\Omega}\right]$ is bounded from $L M_{p, \varphi_{1}}^{\left\{x_{0}\right\}}$ to $L M_{p, \varphi_{2}}^{\left\{x_{0}\right\}}$. Moreover,

$$
\left\|\left[b, T_{\Omega}\right] f\right\|_{L M_{p, \varphi_{2}}^{\left\{x_{0}\right\}}} \lesssim\|b\|_{L C_{p_{2}, \lambda}^{\left\{x_{0}\right)}}\|f\|_{L M_{p, \varphi_{1}}^{\left\{x_{0}\right\}}} .
$$

Proof The statement of Theorem 4.7 follows by Lemma 4.6 and Theorem 4.2 in the same manner as in the proof of Theorem 3.8.

Corollary 4.8 Suppose that $x_{0} \in \mathbb{R}^{n}, \Omega \in L_{s}\left(S^{n-1}\right)$ with $s>1$, is homogeneous of degree zero. Let $1<p<\infty, b \in L C_{p_{2}, \lambda}^{\left\{x_{0}\right\}}\left(\mathbb{R}^{n}\right), \frac{1}{p}=\frac{1}{p_{1}}+\frac{1}{p_{2}}$, and $0 \leq \lambda<\frac{1}{n}$. Let also, for $s^{\prime} \leq p_{1}$, the pair $\left(\varphi_{1}, \varphi_{2}\right)$ satisfy the condition (4.5), and, for $p<s$, the pair $\left(\varphi_{1}, \varphi_{2}\right)$ satisfy the condition (4.6). Then the operator $\left[b, \bar{T}_{\Omega}\right]$ is bounded from $L M_{p, \varphi_{1}}^{\left\{x_{0}\right\}}$ to $L M_{p, \varphi_{2}}^{\left\{x_{0}\right\}}$.

Corollary 4.9 Let $T_{\Omega}$ be a linear operator satisfying condition (1.1) with $\Omega \in L_{s}\left(S^{n-1}\right), s>1$, being homogeneous of degree zero and bounded on $L_{p}\left(\mathbb{R}^{n}\right)$. Suppose $1<p<\infty$ and $b \in$ $B M O\left(\mathbb{R}^{n}\right)$. Let also, for $s^{\prime} \leq p$, the pair $\left(\varphi_{1}, \varphi_{2}\right)$ satisfy the condition

$$
\int_{r}^{\infty}\left(1+\ln \frac{t}{r}\right) \frac{\operatorname{essinf}_{t<\tau<\infty} \varphi_{1}(x, \tau) \tau^{\frac{n}{p}}}{t^{\frac{n}{p}+1}} d t \leq C \varphi_{2}(x, r)
$$

and, for $p<s$, the pair $\left(\varphi_{1}, \varphi_{2}\right)$ satisfy the condition

$$
\int_{r}^{\infty}\left(1+\ln \frac{t}{r}\right) \frac{\operatorname{essinf}_{t<\tau<\infty} \varphi_{1}(x, \tau) \tau^{\frac{n}{p}}}{t^{\frac{n}{p}-\frac{n}{s}+1}} d t \leq C \varphi_{2}(x, r) r^{\frac{n}{s}}
$$

where $C$ does not depend on $x$ and $r$.

Then the operator $\left[b, T_{\Omega}\right]$ is bounded from $M_{p, \varphi_{1}}$ to $M_{p, \varphi_{2}}$. Moreover,

$$
\left\|\left[b, T_{\Omega}\right] f\right\|_{M_{p, \varphi_{2}}} \lesssim\|b\|_{B M O}\|f\|_{M_{p, \varphi_{1}}} .
$$

Corollary 4.10 Suppose that $\Omega \in L_{s}\left(S^{n-1}\right)$ with $s>1$, is homogeneous of degree zero. Let $1<p<\infty$ and $b \in B M O\left(\mathbb{R}^{n}\right)$. Let also, for $s^{\prime} \leq p$, the pair $\left(\varphi_{1}, \varphi_{2}\right)$ satisfy the condition (4.7) and, for $p<s$, the pair $\left(\varphi_{1}, \varphi_{2}\right)$ satisfy the condition (4.8). Then the operator $\left[b, \bar{T}_{\Omega}\right]$ is bounded from $M_{p, \varphi_{1}}$ to $M_{p, \varphi_{2}}$.

Remark 4.11 Note that the boundedness of sublinear operators with rough kernel and its commutator on the generalized central (local) Morrey spaces $L M_{p, \varphi}$ were studied in [38]. Also, in the case $s=\infty$ Corollary 4.8 was proved in [30] and Corollary 4.10 in [33]. 
Authors' contributions

All authors contributed equally to the writing of this paper. All authors read and approved the final manuscript.

\section{Author details}

${ }^{1}$ Department of Mathematical Analysis, Baku State University, Baku, Azerbaijan. ${ }^{2}$ Institute of Mathematics and Mechanics of NAS of Azerbaijan, Baku, Azerbaijan. ${ }^{3}$ Department of Mathematics, Ahi Evran University, Kirsehir, Turkey. ${ }^{4}$ Department of Mathematics, Ankara University, Ankara, Turkey.

\section{Acknowledgements}

The authors would like to express their gratitude to the referees for their very valuable comments and suggestions. The research of $V$ Guliyev was partially supported by the grant of Science Development Foundation under the President of the Republic of Azerbaijan, Grant EIF-2013-9(15)-46/10/1 and by the grant of Ahi Evran University Scientific Research Projects (PYO.FEN.4001.13.012)

Received: 20 September 2014 Accepted: 29 January 2015 Published online: 20 February 2015

\section{References}

1. Soria, F, Weiss, G: A remark on singular integrals and power weights. Indiana Univ. Math. J. 43, 187-204 (1994)

2. Lu, G, Lu, S, Yang, D: Singular integrals and commutators on homogeneous groups. Anal. Math. 28, 103-134 (2002)

3. Ding, Y, Yang, $D, Z$ hou, Z: Boundedness of sublinear operators and commutators on $L^{p, \omega}\left(\mathbb{R}^{n}\right)$. Yokohama Math. J. 46 , 15-27 (1998)

4. Morrey, CB: On the solutions of quasi-linear elliptic partial differential equations. Trans. Am. Math. Soc. 43, 126-166 (1938)

5. Adams, DR: A note on Riesz potentials. Duke Math. J. 42, 765-778 (1975)

6. Chiarenza, F, Frasca, M: Morrey spaces and Hardy-Littlewood maximal function. Rend. Mat. 7, 273-279 (1987)

7. Peetre, J: On the theory of $M_{p, \lambda}$. J. Funct. Anal. 4, 71-87 (1969)

8. Chiarenza, F, Frasca, M, Longo, P: Interior $W^{2, p}$-estimates for nondivergence elliptic equations with discontinuous coefficients. Ric. Mat. 40, 149-168 (1991)

9. Chiarenza, F, Frasca, M, Longo, P: W2,P-Solvability of Dirichlet problem for nondivergence elliptic equations with VMO coefficients. Trans. Am. Math. Soc. 336, 841-853 (1993)

10. Di Fazio, G, Ragusa, MA: Interior estimates in Morrey spaces for strong solutions to nondivergence form equations with discontinuous coefficients. J. Funct. Anal. 112, 241-256 (1993)

11. Di Fazio, G, Palagachev, DK, Ragusa, MA: Global Morrey regularity of strong solutions to the Dirichlet problem for elliptic equations with discontinuous coefficients. J. Funct. Anal. 166, 179-196 (1999)

12. Mizuhara, T: Boundedness of some classical operators on generalized Morrey spaces. In: Igari, S (ed.) Harmonic Analysis. ICM 90 Satellite Proceedings, pp. 183-189. Springer, Tokyo (1991)

13. Nakai, E: Hardy-Littlewood maximal operator, singular integral operators and Riesz potentials on generalized Morrey spaces. Math. Nachr. 166, 95-103 (1994)

14. Samko, N: Weighted Hardy and singular operators in Morrey spaces. J. Math. Anal. Appl. 350(1), 56-72 (2009)

15. Guliyev, VS: Boundedness of the maximal, potential and singular operators in the generalized Morrey spaces J. Inequal. Appl. 2009, Article ID 503948 (2009)

16. Guliyev, VS: Integral operators on function spaces on the homogeneous groups and on domains in $\mathbb{R}^{n}$. Doctor's degree dissertation, Mat. Inst. Steklov, Moscow (1994) 329 pp. (in Russian)

17. Guliyev, VS: Function spaces, Integral Operators and Two Weighted Inequalities on Homogeneous Groups. Some Applications, Cashioglu, Baku (1999) 332 pp. (in Russian)

18. Guliyev, VS: Some properties of the anisotropic Riesz-Bessel potential. Anal. Math. 26, 99-118 (2000)

19. Guliyev, VS, Mustafayev, RC: Integral operators of potential type in spaces of homogeneous type. Dokl. Akad. Nauk, Ross. Akad. Nauk 354, 730-732 (1997)

20. Guliyev, VS, Mustafayev, RC: Fractional integrals in spaces of functions defined on spaces of homogeneous type. Anal. Math. 24, 181-200 (1998)

21. Burenkov, VI, Guliyev, HV Guliyev, VS: Necessary and sufficient conditions for boundedness of the fractional maximal operators in the local Morrey-type spaces. J. Comput. Appl. Math. 208(1), 280-301 (2007)

22. Burenkov, VI, Guliyev, VS: Necessary and sufficient conditions for the boundedness of the Riesz potential in local Morrey-type spaces. Potential Anal. 30(3), 211-249 (2009)

23. Burenkov, V, Gogatishvili, A, Guliyev, VS, Mustafayev, R: Boundedness of the Riesz potential in local Morrey-type spaces. Potential Anal. 35(1), 67-87 (2011)

24. Burenkov, V, Guliyev, VS, Serbetci, A, Tararykova, TV: Necessary and sufficient conditions for the boundedness of genuine singular integral operators in local Morrey type spaces. Eurasian Math. J. 1, 32-53 (2010)

25. Wiener, N: Generalized harmonic analysis. Acta Math. 55, 117-258 (1930)

26. Wiener, N: Tauberian theorems. Ann. Math. 33, 1-100 (1932)

27. Beurling, A: Construction and analysis of some convolution algebras. Ann. Inst. Fourier (Grenoble) 14, 1-32 (1964)

28. Feichtinger, H: An elementary approach to Wiener's third Tauberian theorem on Euclidean $n$-space. In: Symposia Mathematica (Cortona, 1984). Sympos. Math., vol. 29. Academic Press, New York (1987)

29. Alvarez, J, Guzman-Partida, M, Lakey, J: Spaces of bounded $\lambda$-central mean oscillation, Morrey spaces, and $\lambda$-centra Carleson measures. Collect. Math. 51, 1-47 (2000)

30. Guliyev, VS: Local generalized Morrey spaces and singular integrals with rough kernel. Azerb. J. Math. 3(2), 79-94 (2013)

31. Guliyev, VS: Generalized local Morrey spaces and fractional integral operators with rough kernel. J. Math. Sci. (N.Y.) 193(2), 211-227 (2013)

32. Akbulut, A, Guliyev, VS, Mustafayev, R: Boundedness of the maximal operator and singular integral operator in generalized Morrey spaces. Math. Bohem. 137(1), 27-43 (2012)

33. Guliyev, VS, Aliyev, SS, Karaman, T, Shukurov, PS: Boundedness of sublinear operators and commutators on generalized Morrey space. Integral Equ. Oper. Theory 71(3), 327-355 (2011) 
34. Coifman, R, Rochberg, R, Weiss, G: Factorization theorems for Hardy spaces in several variables. Ann. Math. 103(2), 611-635 (1976)

35. Ragusa, MA: Cauchy-Dirichlet problem associated to divergence form parabolic equations. Commun. Contemp. Math. 6(3), 377-393 (2004)

36. Lu, SZ, Yang, DC: The central BMO spaces and Littlewood-Paley operators. Approx. Theory Appl. 11, 72-94 (1995)

37. Guliyev, VS: Generalized weighted Morrey spaces and higher order commutators of sublinear operators. Eurasian Math. J. 3(3), 33-61 (2012)

38. Fan, Y: Boundedness of sublinear operators and their commutators on generalized central Morrey spaces. J. Inequal. Appl. 2013, Article ID 411 (2013)

Submit your manuscript to a SpringerOpen ${ }^{\circ}$ journal and benefit from:

- Convenient online submission

- Rigorous peer review

- Immediate publication on acceptance

- Open access: articles freely available online

- High visibility within the field

Retaining the copyright to your article

Submit your next manuscript at $\gg$ springeropen.com 